\title{
Toll-like receptors regulate $B$ cell cytokine production in patients with diabetes
}

\author{
M. Jagannathan • M. McDonnell • Y. Liang • \\ H. Hasturk • J. Hetzel • D. Rubin • A. Kantarci • \\ T. E. Van Dyke • L. M. Ganley-Leal • B. S. Nikolajczyk
}

Received: 3 November 2009 /Accepted: 24 February 2010/Published online: 11 April 2010

(C) Springer-Verlag 2010

\begin{abstract}
Aims/hypothesis Understanding cellular and molecular events in diabetes mellitus will identify new approaches for therapy. Immune system cells are important modulators of chronic inflammation in diabetes mellitus, but the role of B cells is not adequately studied. The aim of this work was to define the function of B cells in diabetes mellitus patients through focus on $\mathrm{B}$ cell responses to pattern recognition receptors.

Methods We measured expression and function of Toll-like receptors (TLRs) on peripheral blood B cells from diabetes mellitus patients by flow cytometry and multiplexed cytokine analysis. We similarly analysed B cells from non-diabetic donors and periodontal disease patients as comparative cohorts.
\end{abstract}

M. Jagannathan

Department of Pathology, Boston University School of Medicine,

Boston, MA, USA

M. McDonnell $\cdot$ J. Hetzel $\cdot$ D. Rubin

Department of Medicine, Section of Endocrinology,

Evans Biomedical Research Center, Boston Medical Center,

Boston, MA, USA

Y. Liang $\cdot$ L. M. Ganley-Leal

Department of Medicine, Section of Infectious Diseases,

Evans Biomedical Research Center, Boston Medical Center,

Boston, MA, USA

H. Hasturk · A. Kantarci · T. E. Van Dyke

Department of Periodontology and Oral Biology,

Boston University Goldman School of Dental Medicine,

Boston, MA, USA

B. S. Nikolajczyk ( $\bowtie)$

Department of Microbiology,

Boston University School of Medicine,

72 East Concord Street, L-516,

Boston, MA 02118, USA

e-mail: bnikol@bu.edu
Results B cells from diabetes mellitus patients secrete multiple pro-inflammatory cytokines, and IL-8 production is significantly elevated in B cells from diabetic patients compared with those from non-diabetic individuals. These data, plus modest elevation of TLR surface expression, suggest B cell IL-8 hyperproduction is a cytokine-specific outcome of altered TLR function in B cells from diabetes mellitus patients. Altered TLR function is further evidenced by demonstration of an unexpected, albeit modest 'anti-inflammatory' function for TLR4. Importantly, B cells from diabetes mellitus patients fail to secrete IL-10, an anti-inflammatory cytokine implicated in inflammatory disease resolution, under a variety of TLRstimulating conditions. Comparative analyses of B cells from patients with a second chronic inflammatory disease, periodontal disease, indicated that some alterations in B cell TLR function associate specifically with diabetes mellitus.

Conclusions/interpretation Altered TLR function in B cells from diabetes mellitus patients increases inflammation by two mechanisms: elevation of pro-inflammatory IL- 8 and lack of anti-inflammatory/protective IL-10 production.

Keywords B lymphocytes · Cytokines · Diabetes mellitus · Human · Toll-like receptors

\begin{tabular}{ll}
\multicolumn{2}{l}{ Abbreviations } \\
CRP & C-reactive protein \\
GM-CSF & $\begin{array}{l}\text { Granulocyte/macrophage colony stimulating } \\
\text { factor }\end{array}$ \\
LPS & Lipopolysaccharide \\
MFI & Mean fluorescence intensity \\
ND & Non-diabetic \\
ODN & Oligodeoxynucleotide \\
Pam3 & Pam3CSK4 \\
PD & Periodontal disease \\
rLPS & Rhodobacter sphaeroides lipopolysaccharide \\
TLR & Toll-like receptor
\end{tabular}




\section{Introduction}

Chronic inflammation characterises a wide range of disorders, including diabetes mellitus, and associates with diabetes mellitus aetiology and co-morbidities such as cardiovascular disease [1-3]. Inflammation and insulin resistance in diabetes mellitus patients is thought to stem, at least in part, from an ongoing immune response to ligands that include endogenous NEFAs and endotoxin [4-6].

Cells of the innate immune system sense many endogenous ligands and bacterial products through Toll-like receptors (TLRs). TLRs play important roles in diabetes mellitus, as shown both in humans and in animal models of diabetes mellitus. For example, mice with an inactive Tlr4 gene were significantly less prone to diet-induced insulin resistance $[7$, 8]. Likewise, inhibition of TLR2 function in mice exposed to a high-fat diet led to improved insulin sensitivity and decreased activation of pro-inflammatory pathways [9]. Furthermore, polymorphisms in TLRs and in members of TLR-downstream signalling pathways that encode hyperor hypoactive responses predict the development of type 1 and type 2 diabetes [10-12]. Overall, these studies support the idea that TLR2 and TLR4 activities promote diabetes mellitus. It is generally assumed that cells of the myeloid lineage are predominantly responsible for the demonstrated effect of TLRs in diabetes mellitus. However, B cell TLRs have also been recognised as important mediators of innate immune responses in inflammatory diseases $[13,14]$.

Human B cells express multiple TLRs and can produce both pro- and anti-inflammatory cytokines in response to TLR ligands [15-18]. The most commonly studied B cell TLR is TLR9, which mediates B cell response to CpG. $\mathrm{B}$ cells from healthy humans, in contrast to mice, generally express little to no surface TLR2 and TLR4 [15, 16, 19, 20]. However, our recent work demonstrated that B cells from chronic inflammatory disease patients, specifically periodontal disease (PD) patients, have elevated responses to TLR2 and TLR4 ligands compared with B cells from healthy donors $[13,19]$. These studies also showed that cross-talk between TLRs differentially regulates cytokine production by B cells from patients compared with healthy donors. Overall, this work highlighted the complexity and elegant specificity of the B cell response to TLR ligands, and suggested that B cell TLR expression and downstream cytokine production influences the overall milieu in chronic inflammation. Our studies introduced B cell TLRs and TLR-downstream cytokine production as potential players in chronic non-autoimmune inflammatory disease for the first time. Based on high incidence of PD in diabetes mellitus patients, these studies raised the possibility that $\mathrm{B}$ cells may also play roles in the systemic inflammation characterising diabetes mellitus.
The B cell cytokine most commonly implicated in chronic inflammatory diseases is IL-10. IL-10 is generally an anti-inflammatory cytokine that promotes inflammation resolution. B cell IL-10 has specifically been implicated in inflammatory autoimmune diseases characterised by B cell dysfunction. For example, B cells from multiple sclerosis (MS) patients secrete lower levels of IL-10 than B cells from healthy donors, and secretion increases in patients treated palliatively with mitoxantrone. These data suggest that B cell IL-10 production decreases clinical symptoms [21]. IL-10-secreting B cells from healthy donors, but not lupus patients, also block Th1 differentiation [22]. Furthermore, B cell IL-10 is critical for recovery from arthritis and a mouse version of MS (experimental autoimmune encephalitis [23, 24]). Finally, genetic studies have linked elevated IL-10 levels to protection from metabolic syndrome and diabetes mellitus in humans [25], and thus indicate IL-10 can protect against a wide array of chronic inflammatory diseases. Importantly, recent studies demonstrated that B cells specifically require TLRs to produce the IL-10 that blocks T cell-mediated inflammation [26]. Whether similar $\mathrm{B}$ cell TLR responses regulate IL-10 production by $\mathrm{B}$ cells from diabetes mellitus patients is untested.

Differences between TLR expression and function in B cells from chronic inflammatory disease patients $[13,27]$ led us to question how B cell TLRs function in diabetes mellitus patients. We demonstrate that TLR ligands activate B cell cytokine production, most significantly IL-8, in diabetes mellitus vs non-diabetic (ND) donors. Proinflammatory cytokine production is likely to be physiologically bolstered by the complete inability of B cells from diabetes mellitus patients to upregulate IL-10 secretion in response to TLR ligands. Surprisingly, the B cell response to combinations of TLR ligands uncovered further disease-associated changes in TLR function. These results indicate that altered TLR function rather than surface expression levels regulate the B cell contribution to diabetes mellitus. The unexpected anti-inflammatory function of TLR4 coupled with signatures more characteristic of pro-inflammatory cells indicates that fundamental alterations in B cell responses play complex, overall pro-inflammatory roles in systemic inflammation in diabetes mellitus.

\section{Methods}

Cells Human samples were obtained after informed consent under a protocol approved by the Boston University Institutional Review Board and conducted in accordance with the Declaration of Helsinki. We analysed cells from a cross-sectional group of diabetes mellitus patients recruited from the Center for Endocrinology, Diabetes and Nutrition 
at Boston Medical Center. The cohort excluded individuals with common infections $\leq 2$ weeks prior to donation or those with non-diabetes mellitus related inflammatory or auto-immune disease. Diabetes mellitus patients were defined as: (1) under the care of a Center for Diabetes provider; (2) on one or more diabetes medications; and (3) having a diagnosis of diabetes on the clinical problem list. C-reactive protein (CRP) was measured at the time of blood draw as an established biomarker of systemic inflammation in metabolic disease [28]. Approximately $50 \%$ of the diabetes mellitus patients would be considered average risk and several as high risk for cardiovascular disease, as previously defined by CRP [29]. Peripheral blood was collected into heparinised tubes by venous puncture and B cells were purified as published [19]. The majority of contaminating cells in the $>96 \%$ pure $\mathrm{B}$ cell preparations were $\mathrm{T}$ cells; monocyte contamination was undetectable. Table 1 outlines the diabetes mellitus patients $(n=11$; nine with type 2 diabetes, two with type 1 diabetes but lacking autoimmune antibodies) who provided blood B cells for cytokine analyses. B cells were also provided by ND PD patients who had diagnoses of localised aggressive periodontitis [30] but no other known disease. These patients $(n=14)$ were recruited from the Clinical Research Center at Boston University Medical Center (Table 2). A detailed description of PD is published in Shin et al. [19]. Healthy donors had no indication of metabolic disease or PD excepting mild gingivitis $(n=19$; Table 2$)$. None of the participants smoked.

Table 1 Description of diabetes mellitus patients used for cytokine analysis $(n=11$ total)

\begin{tabular}{ll}
\hline Characteristic & Value \\
\hline Age, years (median and range) & $55(38-72)$ \\
$\mathrm{HbA}_{1 \mathrm{c}}, \%$ (median and range) & $8.2(6.0-14.9)$ \\
Duration of diabetes mellitus, & $10(5-32)$ \\
years (median and range) & $32.4(17.0-43.1)$ \\
BMI, $\mathrm{kg} / \mathrm{m}^{2}$ (median and range) & $9(1-164)$ \\
$\mathrm{CRP}{ }^{\mathrm{a}}, \mathrm{mg} / 1$ (median and range) & $72.7^{\mathrm{b}}$ \\
Insulin usage, $n=8$ (percentage of total) & 82 \\
Type 2 diabetes, $n=9$ (percentage of total) & 18 \\
Type 1 diabetes, $n=2$ (percentage of total) & 54.5 \\
Males, $n=6$ (percentage of total) & \\
Race & 54.5 \\
White, $n=6$ (percentage of total) & 45.5 \\
African-American, $n=5$ (percentage of total) &
\end{tabular}

\footnotetext{
${ }^{a}$ Single CRP values available for eight of 11 patients; median is within normal range

${ }^{\mathrm{b}}$ Insulin usage by one type 2 diabetes patient is unknown
}

Flow cytometry For surface staining, $100 \mu \mathrm{l}$ whole blood (coded to avoid bias) was labelled with conjugated antibodies purchased from BD Pharmingen (San Diego, CA, USA: anti-CD3, -CD14, -CD19, -CD23, -CD27, -CD38, -CD69, -CD77 and -RP105) or eBioscience (San Diego, CA, USA: anti-TLR4 and isotype controls). Erythrocytes were lysed with $2 \mathrm{ml} 1 \times$ FACS Lysing Solution for $30 \mathrm{~min}$. Cells were washed with $0.2 \%$ (wt/vol.) BSA/PBS and fixed with $2 \%$ (wt/vol.) paraformaldehyde, then analysed on a FACSCaliber (BD Biosciences, San Jose, CA, USA) with WinMDI software (J. Trotter, The Scripps Institute, Palo Alto, CA, USA). Intracellular staining was completed on mononuclear fractions incubated in medium or with antiIg $\mu$ for $24 \mathrm{~h}$. Brefeldin A (eBioscience) was added at $21 \mathrm{~h}$. Cells were stained with anti-CD14 or anti-CD19 for $20 \mathrm{~min}$ at $4{ }^{\circ} \mathrm{C}$, then washed with $0.2 \%(\mathrm{wt} / \mathrm{vol}$.) $\mathrm{BSA} / \mathrm{PBS}$, and treated with Fixation buffer then $1 \times$ Permeabilisation buffer (eBioscience). Cells were then stained with anti-TNF- $\alpha$, anti-IL-10 (eBioscience) or anti-IL-8 (BD Pharmingen), and washed with $1 \times$ Permeabilisation buffer. Resuspended cells were analysed on a FACScan (BD Biosciences) with CellQuest (BD Biosciences) and FloJo (Tree Star, Ashland, OR, USA) software.

Biochemistry B cells were negatively isolated with magnetic beads (Miltenyi, Auburn, CA, USA) and rested (1 h $37^{\circ} \mathrm{C}$ ) before stimulation. Cultures were 250,000 cells/ $250 \mu \mathrm{l}$ in U-bottom wells. For constitutive cytokine production, B cells were incubated in complete medium (RPMI, 10\% [vol./vol.] heat-inactivated FCS) alone. Alternatively, TLR ligands, or anti-Ig $\mu /$ anti-CD40 were each added to a concentration of $1 \mu \mathrm{g} / \mathrm{ml}$. TLR ligands (Invivogen; San Diego, CA, USA) were: Pam3CSK4 (Pam3; TLR2 ligand), Rhodobacter sphaeroides lipopolysaccharide (LPS) (rLPS; TLR4 ligand [31]), CpG oligodeoxynucleotide (ODN) 2006 (TLR9 ligand) and ultrapure E. coli LPS 0111:B4 (TLR4 ligand). Cells were stimulated for $24 \mathrm{~h}$ prior to cytokine analysis with an Invitrogen (Carlsbad, CA, USA) kit that measured IL-1 $\beta$, IL-2, IL-4, IL-5, IL-6, IL-8, IL-10, IFN- $\gamma$, TNF- $\alpha$ and granulocyte/ macrophage colony stimulating factor (GM-CSF) on a Luminex 200 machine (Luminex, Austin, TX, USA). Chromatin accessibility by real-time PCR (CHART-PCR) was completed as published [32].

Statistics The Mann-Whitney $U$ test was used to compare values between cohorts. Paired non-parametric (Wilcoxon) $t$ tests established significance for cytokine production by variously stimulated B cells from the same individual. $p<0.05$ established significance. Analyses were performed on Prism (GraphPad Software, La Jolla, CA, USA). Sample numbers varied because of the variable number of $\mathrm{B}$ cells isolated per donor. 
Table 2 Description of PD and ND individuals

\begin{tabular}{lll}
\hline Characteristic & PD & ND \\
\hline Age, years (median and range) & $32(20-38)$ & $31(22-52)$ \\
$n$ & 14 & $19^{\mathrm{a}}$ \\
Males, $n(\%)$ & $7(50)$ & $9(47)$ \\
Race, $n(\%)$ & & \\
White & $2(14.3)$ & $6(31.6)$ \\
African-American & $11(78.6)$ & $8(42.1)$ \\
Asian & $1(7.1)$ & $0(0)$ \\
Hispanic & $1(7.1)$ & $1(5.2)$ \\
Unknown & $0(0)$ & $4(31.6)$ \\
\hline
\end{tabular}

${ }^{a}$ Age and sex of two ND individuals are unknown

\section{Results}

$B$ cells from diabetes mellitus patients constitutively secrete $I L-8$ B cells are important sources of multiple cytokines in vivo $[21,23]$. We reasoned that if $\mathrm{B}$ cell cytokines play a role in systemic inflammation in diabetes mellitus, fresh ex vivo B cells from diabetes mellitus vs ND donors should differentially produce cytokines. We analysed constitutive cytokine production by highly purified populations of B cells, devoid of monocytes (Fig. 1a). Additional measures, previously described [13], ensured even small numbers of theoretically contaminating monocytes contributed $<10 \%$ of total measured cytokines in these assays. $B$ cells from diabetes mellitus and ND donors constitutively secreted similar levels of GM-CSF and IL-6 (Fig. 1b). In contrast, B cells from diabetes mellitus vs ND donors constitutively secreted significantly lower levels of TNF- $\alpha$ and the anti-inflammatory cytokine IL-10 (Fig. 1b), but significantly higher levels of the pro-inflammatory cytokine (and neutrophil chemokine) IL-8 (Fig. 1c). Intracellular staining data confirmed that cytokines originated from the $\mathrm{CD}_{1} 9^{+} \mathrm{B}$ cell population (Fig. 1d). Importantly, comparison of intracellular cytokine staining for B cells vs monocytes from the same individuals show that B cells from diabetes
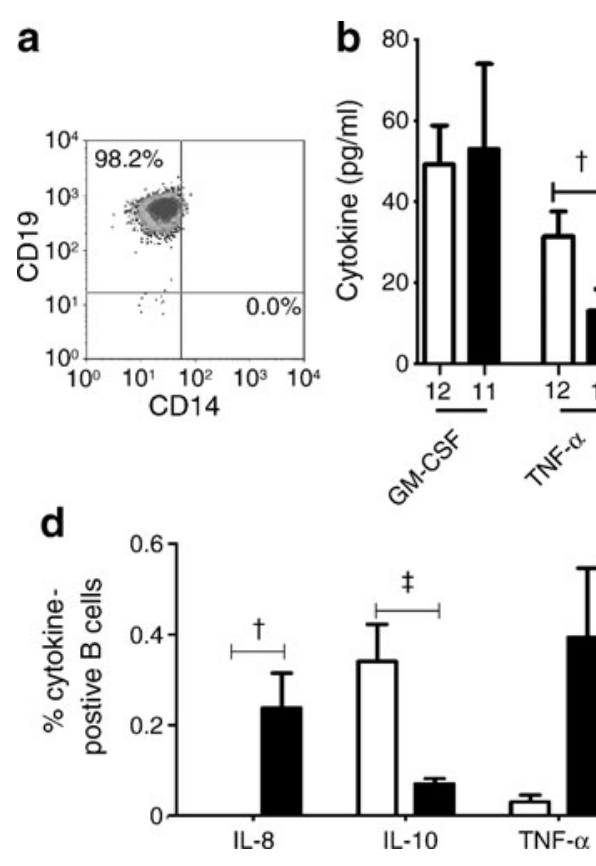

से

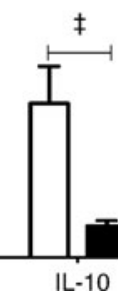

Fig. 1 Purified B cells from diabetes mellitus patients constitutively secrete elevated levels of IL-8 and decreased levels of IL-10. a Representative re-analysis of B cell purity (upper left quadrant) by flow cytometry. Note monocytes were undetectable (bottom right quadrant). b Cytokine production by B cells from ND (white bars) or diabetes mellitus (black bars) donors incubated in medium for $24 \mathrm{~h}$. GM-CSF, TNF- $\alpha$ and IL-10 are quantified on the left $y$-axis; IL-6 is quantified on right $y$-axis. c IL- 8 production by B cells from ND (white bar) or diabetes mellitus (black bar) donors incubated in medium for $24 \mathrm{~h}$. Shown is mean and SEM of the number of samples indicated by the value immediately below each bar. Not all supernatant fractions from all samples were assayed for every cytokine. Significance was determined by Mann-Whitney $U$ test and $p$ values that are significant in comparisons between diabetes mellitus
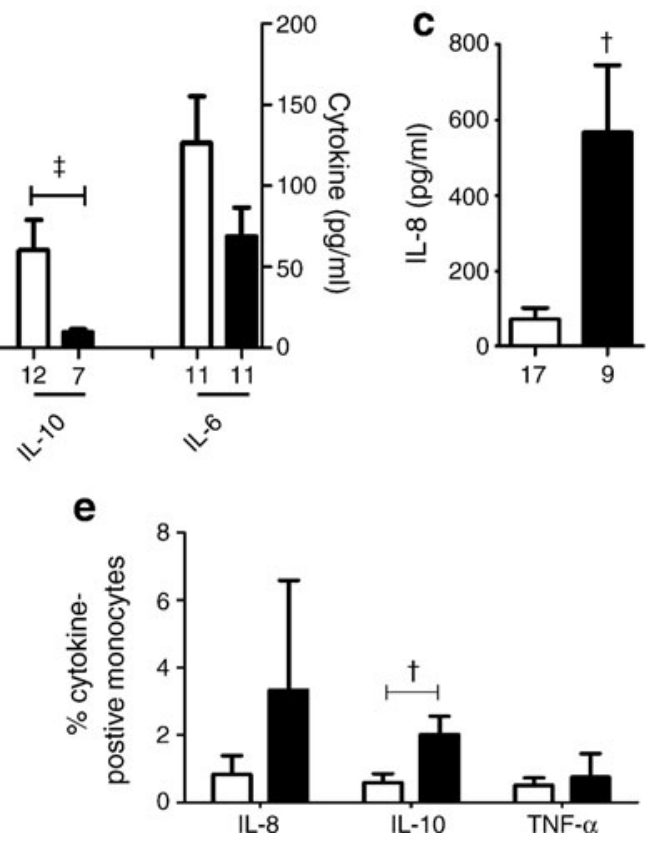

and ND values are shown (b ${ }^{\dagger} p=0.0152,{ }^{\star} p=0.0017$; $\mathbf{c}^{\dagger} p=0.0081$ ). d, e Intracellular cytokine staining in peripheral blood mononuclear cells from ND (white bars) or diabetes mellitus (black bars) donors. d B cells were identified by surface CD19 expression. e Monocytes were identified by surface CD14 expression (absent on B cells). Shown is mean (after subtraction of isotype control) and SD of the percentage of cytokine-positive cells from three donors. All significant differences, calculated by $t$ test, are indicated (d ${ }^{\dagger} p=0.0398,{ }^{\star} p=0.0309$ ). The difference in IL-10 production in panel e approached significance $\left({ }^{\dagger} p=0.0768\right)$. B cell intracellular TNF- $\alpha$ and monocyte intracellular IL8 were highly variable $(p>0.07)$ and hence not definitive. d, e Donors had a diagnosis of type 2 diabetes mellitus and included one black female and two Hispanic males, mean age 56 years. ND donors included two white females and one white male, mean age 47 years 
mellitus patients are uniquely defective for IL-10 production (Fig. 1d, e). For all cytokine analyses, results from patients with type $1(n=2)$ and type $2(n=9)$ diabetes were indistinguishable (data not shown). These data support the conclusion that $\mathrm{B}$ cells from diabetes mellitus patients make a significantly different contribution to the circulating balance of cytokines than B cells from ND donors. The mixed anti-inflammatory (lower TNF- $\alpha$ production) and pro-inflammatory (decreased IL-10 and increased IL- 8 production) signature suggests that $\mathrm{B}$ cells may play an unappreciated role in the chronic inflammation of diabetes mellitus.

A modestly elevated percentage of $B$ cells from diabetes mellitus patients express surface TLR4 Monocytes produce IL-8 in response to ligands that engage TLR2 or TLR4 $[13,20]$. These findings raise the possibility that constitutive B cell IL-8 production (Fig. 1c) may stem from chronic TLR engagement in vivo. However, multiple studies have shown human B cells respond poorly to TLR2 and TLR4 ligands, probably because of low surface expression of their receptors $[16,19,33]$. We therefore speculated diabetes mellitus B cells constitutively secrete high levels of IL-8 owing to elevated expression of surface TLRs that allow increased engagement by endogenous ligands. We first measured surface levels of TLR4 on B cells from diabetes mellitus and ND donors in whole blood as shown in Fig. 2a. The median percentage of TLR4-positive B cells from the diabetes mellitus cohort (Fig. 2b, 27.1\%) was significantly higher $(\sim 60 \%, p=0.024)$ than the median percentage of TLR4-positive B cells from healthy individuals $(16.5 \%)$. The mean fluorescence intensity (MFI), an indication of the number of cell surface TLR4 molecules, was similar between the two cohorts (not shown). This modest increase in the percentage of surface TLR4-positive $\mathrm{B}$ cells in diabetes mellitus patients is consistent with the demonstration that the TLR4 promoter is packaged into an equivalently accessible chromatin structure in B cells from a

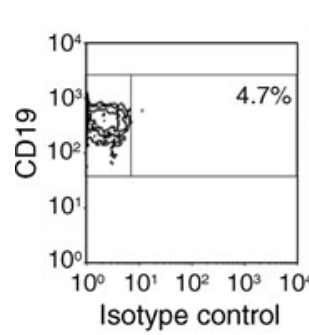

C

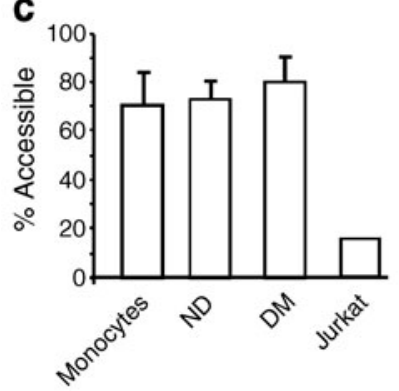

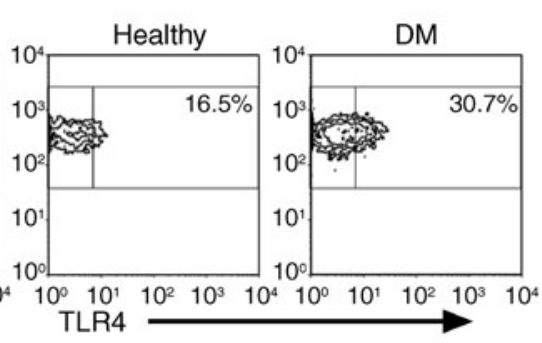

d

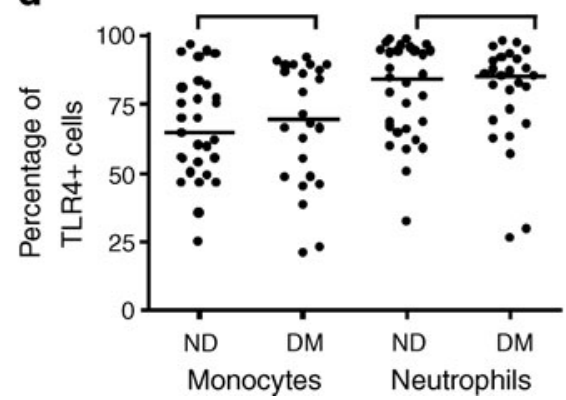

b

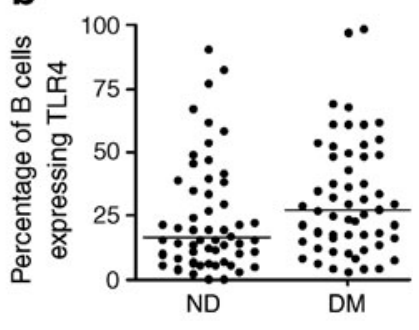

e

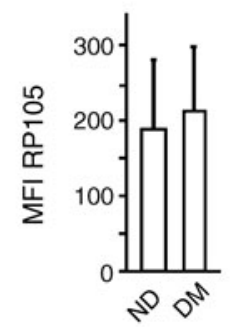

Fig. 2 A modestly elevated percentage of B cells from diabetes mellitus patients express surface TLR4. a A representative flow cytometric analysis of TLR4 expression on the surface of B cells. Isotype control on sample from a representative diabetes mellitus patient is shown in the left panel. Results from TLR4 staining of a representative ND donor and the same diabetes mellitus patient are shown in the middle or right panels, respectively. B cells were identified based on CD19 surface expression ( $y$-axis). Percentage numbers indicate the proportion of B cells that were also TLR4positive. b Composite data showing percentage of TLR4-positive B cells in ND donors and diabetes mellitus patients as indicated. Each point shows analysis of a single sample; horizontal lines show median percentage TLR4-positive B cells for each cohort. The difference (median $=16.45 \%$ or $27.1 \%$ for B cells from ND or diabetes mellitus donors, respectively) was significant $(p<0.05) . n=56$ each for ND and diabetes mellitus samples. c Accessibility of the TLR4 promoter to $2 \mathrm{U}$ micrococcal nuclease in ND monocytes or B cells, or diabetes mellitus B cells as labelled. Human Jurkat $\mathrm{T}$ cells are a negative control. d Percentage TLR4-positive monocytes (left) or neutrophils (right) in peripheral blood of ND or diabetes mellitus donors as indicated. $n=29$ for ND monocytes; $n=24$ for diabetes mellitus monocytes; $n=31$ for ND neutrophils; $n=26$ for diabetes mellitus neutrophils. Horizontal lines shows medians. Differences were insignificant. e RP105 surface expression levels as measured by MFI on $\mathrm{B}$ cells from ND donors or diabetes mellitus patients. Bar shows mean $( \pm \mathrm{SD})$ of ten samples from each cohort. Difference was insignificant $(p>0.1)$. $>95 \%$ of B cells in each cohort expressed surface RP105 (not shown). Statistical significance was determined by Mann-Whitney $U$ test for all comparisons, except panel d, which was analysed by ANOVA 
diabetes mellitus and ND donors (Fig. 2c). Notably, preliminary detailed flow cytometric analyses showed TLR4 was globally upregulated on all circulating B cell subsets ([33] and data not shown). Furthermore, we detected no overall differences in the distribution of $\mathrm{B}$ cell subpopulations in ND vs diabetes mellitus donors for a standard group of human B cell surface markers (not shown). Percentages of TLR4-positive B cells did not correlate with age, BMI, PD or statin use in preliminary analysis of confounding factors (not shown). Importantly, the percentage of TLR4-expressing blood monocytes $\left(\mathrm{CD} 14^{+}\right.$cells) or neutrophils $\left(\mathrm{CD} 14^{\mathrm{low}} \mathrm{CD} 63^{\text {low }} \mathrm{Mac}-1^{\text {high }}\right)$ was similar between diabetes mellitus and ND donors (Fig. 2d), suggesting that modestly increased percentages of TLR4-positive B cells (Fig. 2b) was cell-type limited. Note that the monocyte data contrast with reports showing increased TLR4 expression on purified monocytes from newly diagnosed patients with type 2 diabetes [34], raising the possibility that either diabetic drugs taken by our diabetes mellitus cohort, or activation of monocytes during processing in the cited work may influence monocyte TLR4 levels. Regardless, B cells from diabetes mellitus and ND donors expressed indistinguishable surface levels of RP105, an alternative LPS receptor, as measured by MFI (Fig. 2e) or percentage of positive cells ( $>95 \%$ for both cohorts, not shown). Furthermore, our published analyses showed no difference in the percentage of TLR2-positive B cells from diabetes mellitus and ND donors [20]. These modest/ insignificant changes in surface TLR expression on B cells from diabetes mellitus patients suggest that if constitutive IL-8 production is TLR-dependent, changes in TLR function rather than TLR expression may play a role in diabetes mellitus inflammation.

TLR2 and TLR4 ligands regulate B cell cytokine production To identify diabetes mellitus-associated differences in B cell TLR function, we stimulated purified B cells from diabetes mellitus and ND donors with model TLR ligands and measured cytokine production. Pam3/TLR2 activation of $\mathrm{B}$ cells from diabetes mellitus and ND donors showed that diabetes mellitus B cells produced significantly increased levels of IL-8 (Fig. 3a). The TLR-activated IL-8 levels produced by B cells were in the same range as levels of IL-8 production reported for monocytes [35]. B cells from diabetic patients, but not healthy donors, also produced IL-8 in response to anti-Ig $\mu$ stimulation (Fig. 3b), indicating diabetes mellitus B cells may be abnormally poised to produce IL-8. TLR2 ligand significantly increased production of multiple cytokines by diabetes mellitus B cells (Table 3), but this increase was not greater than that observed for B cells from ND donors (Fig. 3c, d), and increased IL-10 production (to $\sim 10 \mathrm{pg} / \mathrm{ml}$ ) was quantitatively meagre. These results are consistent with published data showing ND B cells respond modestly to TLR2 stimulation [33]. In addition, B cells from diabetes mellitus vs ND donors responded to the TLR4 ligand rLPS by producing elevated levels of IL-8. By contrast, B cells from the two cohorts produced similar levels of other cytokines tested (Fig. 3a, c, d). Under all conditions, B cells from diabetes mellitus and ND donors produced undetectable levels of IL-2, -4, -5 and IFN- $\gamma$ (not shown), consistent with the interpretation that $\mathrm{T}$ cells, the major contaminant of
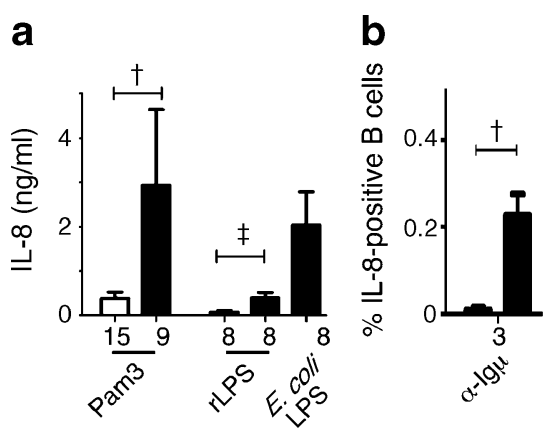

C

Fig. 3 IL-8 is significantly increased in B cells from diabetes mellitus patients vs ND donors responding to TLR2 ligand (Pam3). Purified $\mathrm{B}$ cells from diabetes mellitus (black bars) or ND (white bars) donors were incubated for $24 \mathrm{~h}$ in medium alone or with TLR4 ligand (rLPS or E. coli LPS) or TLR2 ligand (Pam3) as indicated. Samples were analysed for production of IL-8 (a, b), TNF- $\alpha$ (c), GM-CSF (d), or cytokines indicated below the $x$-axis (e) in B cell responding to $E$. coli LPS. Number of samples is indicated by the value immediately below each bar. a, c, d Cytokines measured by multiplex protein analyses. b Percentage of CD19+ B cells that were positive for intracellular IL-8, as compared with isotype control upon stimulation with anti-Ig $\mu$. Anti-Ig $\mu$ induced low but somewhat variable levels of IL-10 and

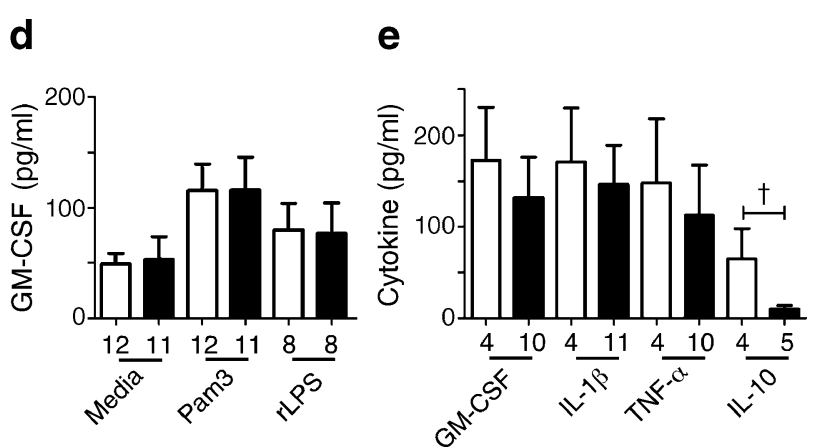

TNF- $\alpha$ in B cell parallel analyses (not shown). Bars show mean and SEM. Medium controls for panel a are shown in Fig. 1c. $p$ values calculated by Mann-Whitney $U$ test are shown above bars for samples that were significantly different $\left(p<0.05\right.$; a ${ }^{\dagger} p=0.0123 ;{ }^{\star} p=0.0381$; $\left.\mathbf{b}^{\dagger} p=0.0124\right)$. All other comparisons were statistically insignificant $(p>0.05)$, although differences in IL-10 production in panel e approached significance $\left({ }^{\dagger} p=0.0635\right)$. Comparison between medium and TLR-stimulated cytokine production indicated significant upregulation for many cytokines in B cells responding to TLR ligand; these values are shown for diabetes mellitus and ND B cell samples in Table 3 
Table 3 Cytokine production by TLR-stimulated B cells

\begin{tabular}{|c|c|c|c|c|}
\hline \multirow[t]{2}{*}{ Cytokine } & \multicolumn{4}{|l|}{ Stimulus } \\
\hline & Medium & Pam3 & rLPS & $\mathrm{CpG}$ \\
\hline \multicolumn{5}{|l|}{ GM-CSF } \\
\hline $\mathrm{T} 2 \mathrm{D}$ mean & 69.3 & 99.7 & 77.8 & 84.4 \\
\hline$p$ value & - & $0.0051^{*}$ & $0.0207^{*}$ & $0.002 *$ \\
\hline$n^{\mathrm{a}}$ & 11 & 11 & 8 & 11 \\
\hline ND mean & 43.6 & 108.1 & 71.4 & 120.8 \\
\hline$p$ value & - & $0.0050^{*}$ & 0.0781 & 0.0625 \\
\hline$n^{\mathrm{a}}$ & 14 & 14 & 9 & 7 \\
\hline \multicolumn{5}{|l|}{ IL-10 } \\
\hline T2D mean & 4.0 & 10.4 & 5.1 & 14.0 \\
\hline$p$ value & - & $0.0300 *$ & 0.2000 & 0.0754 \\
\hline$n^{\mathrm{a}}$ & 7 & 7 & 4 & 7 \\
\hline ND mean & 24.2 & 71.0 & 34.4 & 110.5 \\
\hline$p$ value & - & $0.0025^{*}$ & 0.1563 & $0.0313^{*}$ \\
\hline$n^{\mathrm{a}}$ & 12 & 12 & 8 & 7 \\
\hline \multicolumn{5}{|l|}{ IL-6 } \\
\hline $\mathrm{T} 2 \mathrm{D}$ mean & 68.9 & 541.5 & 153.2 & 580 \\
\hline$p$ value & - & $0.0010^{*}$ & $0.0078^{*}$ & $0.0049^{*}$ \\
\hline$n^{\mathrm{a}}$ & 11 & 11 & 8 & 11 \\
\hline ND mean & 325.5 & $1,622.5$ & 989.7 & 715.5 \\
\hline$p$ value & - & $0.0020^{*}$ & $0.0078^{*}$ & $0.0313^{*}$ \\
\hline$n^{\mathrm{a}}$ & 14 & 12 & 9 & 7 \\
\hline \multicolumn{5}{|l|}{ TNF- $\alpha$} \\
\hline $\mathrm{T} 2 \mathrm{D}$ mean & 16.5 & 86.5 & 28.5 & 116.8 \\
\hline$p$ value & - & $0.0078^{*}$ & 0.2500 & 0.0781 \\
\hline$n^{\mathrm{a}}$ & 8 & 10 & 7 & 10 \\
\hline ND mean & 40.2 & 87.4 & 65.6 & 193.0 \\
\hline$p$ value & - & $0.0025^{*}$ & 0.0781 & 0.0625 \\
\hline$n^{\mathrm{a}}$ & 14 & 14 & 9 & 7 \\
\hline
\end{tabular}

All values for cytokines are in $\mathrm{pg} / \mathrm{ml}$. For IL-10, values from T2D B cells approached the level of assay sensitivity

${ }^{a}$ Number of donors in mean value

${ }^{*} p<0.05$ in TLR-stimulated with medium control for B cells from the same cohort (diabetes mellitus or ND) by paired $t$ test

B cell preparations $(<2 \%)$ insignificantly contributed to TLR-induced cytokine production. Overall, these data support the idea that elevated constitutive IL- 8 production shown for diabetes mellitus B cells in Fig. 1c could result from in vivo hyperresponse to TLR2 and/or TLR4 ligand, in the absence of highly elevated surface TLR expression (Fig. 2b and Noronha et al. [20]).

To further test the possibility that IL-8 is uniquely poised for TLR-mediated activation in diabetes mellitus B cells, we expanded our analysis of TLR-responsive cytokines by first measuring cytokine production in B cells responding to the prototypic TLR4 ligand E. coli LPS. Although B cells from diabetes mellitus donors produce significant amounts of
IL-8 in response to $E$. coli LPS ( $>2 \mathrm{ng} / \mathrm{ml}$ on average; Fig. 3a), B cells from both diabetes mellitus and ND donors responded similarly to $E$. coli LPS (Fig. 3e) as indicated by quantification of GM-CSF, IL-1 $\beta$ and TNF- $\alpha$ production. Note that high variability in values from ND donor B cells prevented direct comparison with IL-8 production by ND B cells (not shown). The overall response of all B cells to $E$. coli LPS (not shown) was relatively modest (i.e. $<200 \mathrm{pg} / \mathrm{ml}$ cytokine, Fig. 3e), consistent with the general dogma that human B cells fail to respond vigorously to this TLR4 ligand. This lack of response is probably due to, at least in part, the lack of the LPS delivery molecule CD14 on the B cell surface (data not shown). Importantly, E. coli LPS failed to activate even modest production of IL-10 by B cells from diabetes mellitus donors, instead stimulating levels that were lower than levels produced by ND B cells (Fig. 3e). We conclude that diabetes mellitus-associated differences in B cell TLR function specifically increase IL- 8 production (Fig. 3a), but are not 'generally' pro-inflammatory as measured by diabetogenic cytokines such as TNF- $\alpha$ and IL-1 $\beta$ [13]. Furthermore, the data suggest that B cells from diabetes mellitus patients are deficient in anti-inflammatory IL-10 production.

TLR engagement can activate B cell IL-10 production, which is critical for inflammatory disease resolution [26]. To more rigorously test the possibility that $\mathrm{B}$ cells from diabetes mellitus patients fail to produce IL-10 in response to TLR engagement, we measured IL-10 production by diabetes mellitus and ND B cells responding to a more comprehensive panel of TLR ligands. In contrast to B cells from ND donors, B cells from diabetic patients fail to activate even modest IL-10 production $(>20 \mathrm{pg} / \mathrm{ml})$ in response to TLR2 (Pam3) or TLR4 (rLPS) ligand (Fig. 4a).
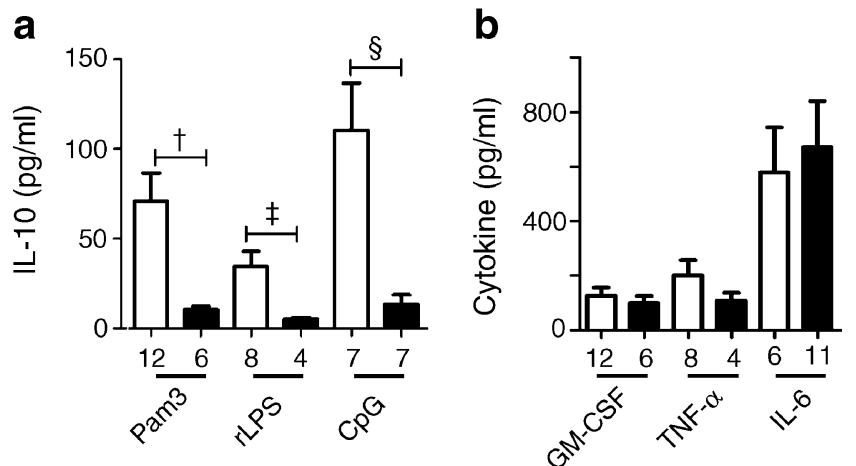

Fig. 4 TLR ligands fail to activate IL-10 production by B cells from diabetes mellitus patients. B cells from ND (white bars) or diabetes mellitus (black bars) donors were incubated for $24 \mathrm{~h}$. with stimuli as indicated. a IL-10 production. b Positive control showing B cells from diabetes mellitus patients are capable of producing cytokines in response to the TLR9 ligand CpG ODN 2006. Shown is mean and SEM of the number of samples indicated immediately below each bar. $p$ values calculated by Mann-Whitney $U$ test are shown above bars for samples that were significantly $(p<0.05)$ different between ND and diabetes mellitus cohorts $\left(\mathbf{a}^{\dagger} p=0.0023 ;{ }^{\ddagger} p=0.0040 ;{ }^{\S} p=0.0012\right)$ 
The latter finding is consistent with decreased IL-10 response in diabetes mellitus vs ND B cells stimulated by E. coli LPS (Fig. 3e). More surprisingly, B cells from diabetes mellitus patients fail to produce IL-10 in response to $\mathrm{CpG}$, a TLR9 ligand known to activate ND B cells (Fig. 4a). As a control, we confirmed that $\mathrm{CpG}$ equivalently activates B cells from diabetes mellitus and ND donors to produce GM-CSF, TNF- $\alpha$ and IL-6, and that CpGtriggered pathways that activate cytokine production are fundamentally intact in B cells from diabetes mellitus patients (Fig. $4 \mathrm{~b}$ and Table 3). These data support one of two possible interpretations: TLR engagement fails to activate IL-10 production, or TLR engagement actively represses IL-10 production by B cells from diabetes mellitus donors. Our preliminary data showing IL-10 is not produced by B cells from diabetes mellitus patients responding to anti-Ig $\mu$ plus $\operatorname{CD} 40$ ( $n=6$; not shown) support the possibility of TLR-independent defects in IL-10 activation in these cells.

$T N F-\alpha$ is uniquely regulated by TLR4 engagement in $B$ cells from diabetes mellitus patients Many endogenous and pathogen-associated pro-inflammatory ligands activate innate immune system cells through combinations of TLR2 and TLR4 ligands [36], or ligands that may be able engage either TLR2 or TLR4, such as the fatty acid palmitate [5, 37]. These findings raise the possibility that differences in TLR4 function play an important role in responses to more complex ligands in B cells from diabetes mellitus patients. To model $\mathrm{B}$ cell interactions with complex ligands and to further identify alterations in TLR function in B cells from diabetes mellitus patients, we co-stimulated cells with Pam3 and rLPS to simultaneously activate TLR2 and TLR4. We then compared cytokine production in B cells stimulated with Pam3 alone vs the two-ligand combination. For some cytokines, rLPS (TLR4 ligand) significantly decreased Pam3 (TLR2)-mediated cytokine production by B cells from both diabetes mellitus and ND donors (GM-CSF; IL-6; Fig. 5a, c, g; note that differences in panel e trended toward significance). However, rLPS significantly decreased TLR2-mediated TNF- $\alpha$ production in B cells from diabetes mellitus patients, but failed to consistently alter TNF- $\alpha$ production by B cells from ND donors (Fig. $5 \mathrm{~d}, \mathrm{~h}$ ). Importantly, B cells from diabetes mellitus patients fail to secrete IL-10 even in the presence of dual stimuli, further supporting the conclusion that IL-10 production is uncoupled from TLR-downstream pathways only in B cells from diabetes mellitus patients (Fig. 5b, f). The quantitative decrease in TNF- $\alpha$ production by B cells from diabetes mellitus patients caused by TLR4 engagement is modest ( $\sim 30 \%$ decrease). Nonetheless, these findings support the conclusion that TLR4 function is fundamentally altered in B cells from diabetes mellitus patients. Furthermore, the
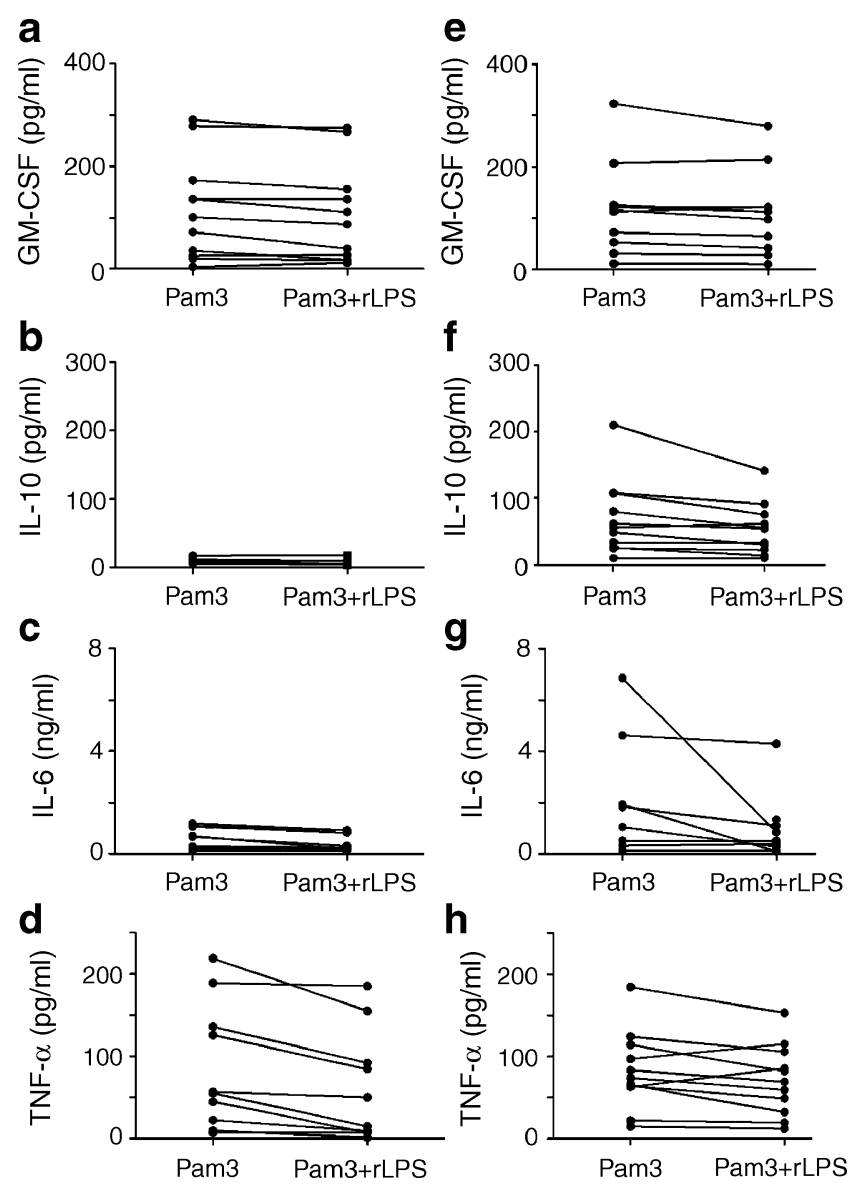

Fig. 5 TLR4 engagement decreases TLR2-mediated TNF- $\alpha$ production by $\mathrm{B}$ cells from diabetes mellitus but not ND donors. Cytokine production by $\mathrm{B}$ cells from diabetes mellitus patients (a-d) or ND donors (e-h) stimulated with the TLR2 ligand Pam3 alone or in combination with the TLR4 ligand rLPS. A line connects values from each donor sample. Significance was determined by a non-parametric paired two-tailed $t$ test. a $p=0.0195 ; \mathbf{b ~ N S}$; c $p=0.0010$; d $p=0.0039$; f $p=0.0098 ; \mathbf{g} p=0.0391 ; \mathbf{h}$ NS. Difference in panel $\mathbf{e}$ approached significance $(p=0.0592)$. Results are from ten diabetes mellitus or 11 ND donor samples

data reveal the specificity by which altered TLR function regulates B cells.

The TLR ligand response of $B$ cells from diabetes mellitus patients is disease-influenced $\mathrm{B}$ cells from multiple chronic inflammatory diseases, including PD, secrete cytokines in response to TLR engagement [13]. Because many diabetes mellitus patients have confounding $\mathrm{PD}$, we asked whether responses of $\mathrm{B}$ cells from diabetic and ND PD patients are similar. Although TLR-induced production of some cytokines by B cells from diabetic vs PD patients was statistically indistinguishable (Fig. 6a, c; GM-CSF and IL-6, respectively), only B cells from PD patients produced IL-10 in response to all stimuli tested (Fig. 6b). Furthermore, IL-8 responses to TLR4 and TLR9 ligand were quantitatively lower in B cells from diabetes mellitus 

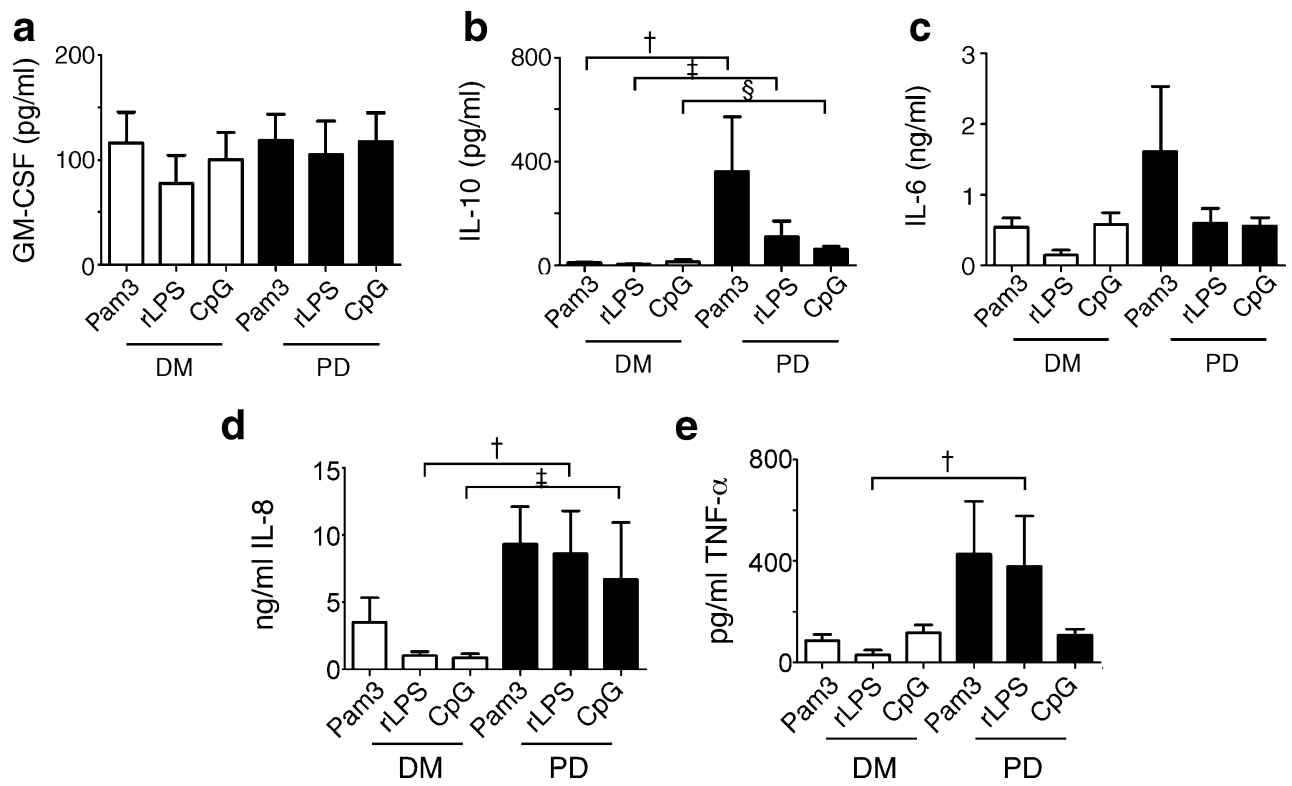

Fig. 6 Disease influences TLR-mediated B cell cytokine production. Comparison of cytokine production by B cells from diabetes mellitus (white bars) or PD (black bars) patients stimulated by the single TLR ligand indicated on the $x$-axis. a GM-CSF; b IL-10; c IL-6; d IL-8; e TNF- $\alpha$. Bars show mean and SEM. Significant differences between values for the two patients cohorts were determined by non-parametric

compared with PD patients (Fig. 6d). Finally, B cells from $\mathrm{PD}$ vs diabetes mellitus patients produce significantly more TNF- $\alpha$ in response to TLR4 ligand (Fig. 6e). The TNF- $\alpha$ results are consistent with the demonstration that TLR4 ligand decreases TLR2-stimulated TNF- $\alpha$ production by B cells from diabetes mellitus (Fig. 5) but not PD [13] donors. Furthermore, the overall increase in pro-inflammatory responses of B cells from PD patients supports the probability that the differences in the age of the cohorts (PD and ND median age is indistinguishable but diabetes mellitus patients are significantly older; Tables 1 and 2) cannot explain all pro-inflammatory changes in patient $\mathrm{B}$ cells. Finally, differences in the subcategory of PD in our defined PD cohort vs our periodontally undefined diabetes mellitus cohort may underlie differences in B cell cytokine production. We conclude that disease pathology influences the $\mathrm{B}$ cell response to TLR ligands. These data further show that lack of B cell IL-10 production is specific to diabetes mellitus.

\section{Discussion}

Taken together, our data indicate that altered TLR function in B cells from diabetes mellitus patients affects cytokine production, and highlights the elegant specificity of TLRmediated outcomes of $\mathrm{B}$ cell activation in disease. two-tailed $t$ tests and are indicated by $p$ values listed above some graphs (b ${ }^{\dagger} p=0.0076,{ }^{\star} p=0.040,{ }^{\circledR} p=0.014$; d ${ }^{\dagger} p=0.034,{ }^{\ddagger} p=0.040$; e ${ }^{\dagger} p=0.019$. All other differences between B cells from the two cohorts stimulated with the same ligand were statistically insignificant $(p>0.05)$

Importantly, surface TLR expression may not be the best predictor of B cell cytokine production in diabetes mellitus. The final outcomes of TLR-activated cytokine production by diabetes mellitus $\mathrm{B}$ cells are, on balance, proinflammatory: increased IL- 8 and decreased IL-10, which may override quantitatively small decreases in TNF- $\alpha$. Although the precise ratio of pro- to anti-inflammatory cytokines required to promote chronic inflammation is unknown, our data clearly indicate that the B cell contribution to the overall ratio is significantly altered in diabetes mellitus and may, for at least IL- 8 and IL-10, rival the importance of monocytes as cytokine producers. The complex contribution of B cells and B cell TLRs to diabetes mellitus inflammation is reminiscent of the mixed contribution of T cells to inflammation in diet-induced obesity, in which $\mathrm{CD} 8 \mathrm{~T}$ cells promote pathology, but regulatory T cells block pathology [38-40].

Elevated IL- 8 production by B cells from diabetes mellitus patients was unexpected, but may link B cells to disease pathogenesis based on several studies implicating IL-8 in diabetes mellitus. IL-8 levels are elevated in serum and in the adipose-associated stromal vascular fraction of diabetes mellitus patients, but the cellular source of IL-8 was not identified by these studies [41, 42]. B cell IL-8 may also play a role in IL-8-associated complications of diabetes mellitus, such as vascular disease [43]. This possibility is further supported by data showing palliative treatments that decrease IL-8 levels in vivo appear to have 
benefits for insulin resistance and cardiovascular risk factors [44]. Taken together, these studies suggest that elevated IL- 8 production by B cells from diabetes mellitus patients may have important implications in diabetes pathogenesis. The concomitant decrease in B cell IL-10 production undoubtedly skews the pro- to antiinflammatory cytokine ratio even further to promote an inflammatory milieu. Although B cells produce lower levels of TNF- $\alpha$ either constitutively or in response to TLR4 ligand (following TLR2 stimulation), the absolute changes in TNF- $\alpha$ production are modest in both assays. Therefore we predict these results hold value as indicators of altered TLR function in diabetes mellitus B cells, but may not identify critical quantitative changes in this diabetes-linked cytokine [45]. The simplistic dogma that TLR-mediated nuclear factor kappa B translocation activates cytokines must be significantly refined to facilitate the design of new diabetes mellitus treatments that exploit knowledge of the highly specific mechanisms that control TLR-activated cytokine production.

Clinical treatments that attempt to decrease inflammation in diabetes mellitus patients have had variable results [4648], perhaps caused in part by a lack of appreciation of nonconventional functions of B cells and B cell TLRs defined herein. Apart from the role B cells may play in diabetes mellitus, B cells certainly play significant roles in vaccine responses. Therefore, the current push to exploit TLR ligands in vaccine adjuvants, some of which will inevitably be used in the growing population of diabetes mellitus patients, makes understanding the responses of these patient $\mathrm{B}$ cells and changes in B cell TLR function more broadly important. Both treatment and vaccine strategies must take into account the net effect of TLR action, including unexpected sources of TLR activity, to most effectively harness the promise of immune system modulation in chronic inflammatory disease patients.

\begin{abstract}
Acknowledgements We thank A. Marshak-Rothstein, R. Corley, M. Clare-Salzler and W. Harnett for manuscript critiques. C. Apovian and A. Bourland generously provided samples for intracellular cytokine analyses. We thank M. Rarick and P. Skolnik from the Center for HIV/AIDS at Boston University Medical Center for use and technical expertise with the multiplex analyser, and G. Denis at the flow core facility at Boston University School of Medicine for expertise in intracellular staining. This work was supported by R01 AI54611 and a Research Grant from the American Diabetes Association (B. S. Nikolajczyk), the Evans Medical Foundation and The Broad Medical Foundation (L. M. Ganley-Leal), DE018917 (H. Hasturk) and DE15566 (T. E. Van Dyke, A. Kantarci and H. Hasturk).
\end{abstract}

Duality of interest The authors declare that there is no duality of interest associated with this manuscript.

\section{References}

1. King GL (2008) The role of inflammatory cytokines in diabetes and its complications. J Periodontol 79:1527-1534

2. Duncan BB, Schmidt MI, Pankow JS et al (2003) Low-grade systemic inflammation and the development of type 2 diabetes: the atherosclerosis risk in communities study. Diabetes 52:17991805

3. Spranger J, Kroke A, Mohlig M et al (2003) Inflammatory cytokines and the risk to develop type 2 diabetes: results of the prospective population-based European Prospective Investigation into Cancer and Nutrition (EPIC)-Potsdam Study. Diabetes 52:812-817

4. Creely SJ, McTernan PG, Kusminski CM et al (2007) Lipopolysaccharide activates an innate immune system response in human adipose tissue in obesity and type 2 diabetes. Am J Physiol Endocrinol Metab 292:E740-E747

5. Shi H, Kokoeva MV, Inouye K, Tzameli I, Yin H, Flier JS (2006) TLR4 links innate immunity and fatty acid-induced insulin resistance. J Clin Invest 116:3015-3025

6. Page RC (1991) The role of inflammatory mediators in the pathogenesis of periodontal disease. J Periodontal Res 26:230 242

7. Poggi M, Bastelica D, Gual P et al (2007) C3H/HeJ mice carrying a Toll-like receptor 4 mutation are protected against the development of insulin resistance in white adipose tissue in response to a high-fat diet. Diabetologia 50:1267-1276

8. Tsukumo DM, Carvalho-Filho MA, Carvalheira JB et al (2007) Loss-of-function mutation in Toll-like receptor 4 prevents diet-induced obesity and insulin resistance. Diabetes 56:1986-1998

9. Caricilli AM, Nascimento PH, Pauli JR et al (2008) Inhibition of toll-like receptor 2 expression improves insulin sensitivity and signaling in muscle and white adipose tissue of mice fed a high-fat diet. J Endocrinol 199:399-406

10. Bagarolli RA, Saad MJ, Saad ST (2010) Toll-like receptor 4 and inducible nitric oxide synthase gene polymorphisms are associated with type 2 diabetes. J Diabetes Complications. doi:10.1016/j. jdiacomp.2009.03.003

11. Kolz M, Baumert J, Muller M et al (2008) Association between variations in the TLR4 gene and incident type 2 diabetes is modified by the ratio of total cholesterol to HDL-cholesterol. BMC Med Genet 9:9

12. Park Y, Park S, Yoo E, Kim D, Shin H (2004) Association of the polymorphism for Toll-like receptor 2 with type 1 diabetes susceptibility. Ann N Y Acad Sci 1037:170-174

13. Jagannathan M, Hasturk H, Liang Y et al (2009) Toll-like receptor cross talk specifically regulates cytokine production by B cells from chronic inflammatory disease patients. J Immunol 183:7461-7470

14. Leadbetter EA, Rifkin IR, Hohlbaum AM, Beaudette BC, Shlomchik MJ, Marshak-Rothstein A (2002) Chromatin-IgG complexes activate B cells by dual engagement of IgM and Toll-like receptors. Nature 416:603-607

15. Bourke E, Bosisio D, Golay J, Polentarutti N, Mantovani A (2003) The toll-like receptor repertoire of human B lymphocytes: inducible and selective expression of TLR9 and TLR10 in normal and transformed cells. Blood 102:956-963

16. Hornung V, Rothenfusser S, Britsch S et al (2002) Quantitative expression of Toll-like receptor 1-10 mRNA in cellular subsets of human peripheral blood mononuclear cells and sensitivity to $\mathrm{CpG}$ oligodeoxynucleotides. J Immunol 168:4531-4537 
17. Duddy ME, Alter A, Bar-Or A (2004) Distinct profiles of human B cell effector cytokines: a role in immune regulation? J Immunol 172:3422-3427

18. Hanten JA, Vasilakos JP, Riter CL et al (2008) Comparison of human B cell activation by TLR7 and TLR9 agonists. BMC Immunol 9:39

19. Shin H, Zhang Y, Jagannathan M et al (2009) B cells from periodontal disease patients express surface Toll-like receptor 4. J Leukoc Biol 85:648-655

20. Noronha AM, Liang Y, Hetzel JT et al (2009) Hyperactivated $\mathrm{B}$ cells in human inflammatory bowel disease. J Leukoc Biol 86:1007-1016

21. Duddy M, Niino M, Adatia F et al (2007) Distinct effector cytokine profiles of memory and naive human B cell subsets and implication in multiple sclerosis. J Immunol 178:6092-6099

22. Blair PA, Norena LY, Flores-Borja F et al (2010) CD19(+)CD24 (hi)CD38(hi) B cells exhibit regulatory capacity in healthy individuals but are functionally impaired in systemic lupus erythematosus patients. Immunity 32:129-140

23. Fillatreau S, Sweenie CH, McGeachy MJ, Gray D, Anderton SM (2002) B cells regulate autoimmunity by provision of IL-10. Nat Immunol 3:944-950

24. Mauri C, Feldmann M, Williams RO (2003) Down-regulation of Th1-mediated pathology in experimental arthritis by stimulation of the Th2 arm of the immune response. Arthritis Rheum 48:839-845

25. van Exel E, Gussekloo J, de Craen AJ, Frolich M, Bootsma-Van Der Wiel A, Westendorp RG (2002) Low production capacity of interleukin-10 associates with the metabolic syndrome and type 2 diabetes: the Leiden 85-Plus Study. Diabetes 51:1088-1092

26. Lampropoulou V, Hoehlig K, Roch T et al (2008) TLR-activated B cells suppress $\mathrm{T}$ cell-mediated autoimmunity. J Immunol $180: 4763-4773$

27. Mealey BL, Ocampo GL (2007) Diabetes mellitus and periodontal disease. Periodontol 2000 44:127-153

28. Freeman DJ, Norrie J, Caslake MJ et al (2002) C-reactive protein is an independent predictor of risk for the development of diabetes in the West of Scotland Coronary Prevention Study. Diabetes 51:1596-1600

29. Rutter MK, Meigs JB, Sullivan LM, D'Agostino RB Sr, Wilson PW (2004) C-reactive protein, the metabolic syndrome, and prediction of cardiovascular events in the Framingham Offspring Study. Circulation 110:380-385

30. Armitage GC (1999) Development of a classification system for periodontal diseases and conditions. Ann Periodontol 4:1-6

31. Golenbock DT, Hampton RY, Qureshi N, Takayama K, Raetz CR (1991) Lipid A-like molecules that antagonize the effects of endotoxins on human monocytes. J Biol Chem 266:19490-19498

32. Rao S, Procko E, Shannon MF (2001) Chromatin remodeling, measured by a novel real-time polymerase chain reaction assay, across the proximal promoter region of the IL-2 gene. J Immunol 167:4494-4503

33. Ganley-Leal LM, Liu X, Wetzler LM (2006) Toll-like receptor 2mediated human B cell differentiation. Clin Immunol 120:272-284
34. Dasu MR, Devaraj S, Park S, Jialal I (2010) Increased Tolllike receptor activation and TLR ligands in recently diagnosed type 2 diabetes subjects. Diabetes Care. doi:10.2337/dc091799

35. Marks DJ, Harbord MW, MacAllister R et al (2006) Defective acute inflammation in Crohn's disease: a clinical investigation. Lancet 367:668-678

36. Darveau RP, Pham TT, Lemley K et al (2004) Porphyromonas gingivalis lipopolysaccharide contains multiple lipid A species that functionally interact with both toll-like receptors 2 and 4 . Infect Immun 72:5041-5051

37. Senn JJ (2006) Toll-like receptor-2 is essential for the development of palmitate-induced insulin resistance in myotubes. J Biol Chem 281:26865-26875

38. Winer S, Chan Y, Paltser G et al (2009) Normalization of obesityassociated insulin resistance through immunotherapy. Nat Med 15:921-929

39. Feuerer M, Herrero L, Cipolletta D et al (2009) Lean, but not obese, fat is enriched for a unique population of regulatory $\mathrm{T}$ cells that affect metabolic parameters. Nat Med 15:930-939

40. Nishimura S, Manabe I, Nagasaki M et al (2009) $\mathrm{CD} 8^{+}$effector $\mathrm{T}$ cells contribute to macrophage recruitment and adipose tissue inflammation in obesity. Nat Med 15:914-920

41. Herder C, Haastert B, Muller-Scholze S et al (2005) Association of systemic chemokine concentrations with impaired glucose tolerance and type 2 diabetes: results from the Cooperative Health Research in the Region of Augsburg Survey S4 (KORA S4). Diabetes 54(Suppl 2):S11-S17

42. Fain JN (2006) Release of interleukins and other inflammatory cytokines by human adipose tissue is enhanced in obesity and primarily due to the nonfat cells. Vitam Horm 74:443-477

43. Qi X, Li J, Gu J, Li S, Dang Y, Wang T (2003) Plasma levels of IL-8 predict early complications in patients with coronary heart disease after percutaneous coronary intervention. Jpn Heart J 44:451-461

44. Bruun JM, Pedersen SB, Richelsen B (2000) Interleukin-8 production in human adipose tissue. inhibitory effects of anti-diabetic compounds, the thiazolidinedione ciglitazone and the biguanide metformin. Horm Metab Res 32:537-541

45. Uysal KT, Wiesbrock SM, Marino MW, Hotamisligil GS (1997) Protection from obesity-induced insulin resistance in mice lacking TNF-alpha function. Nature 389:610-614

46. Larsen CM, Faulenbach M, Vaag A et al (2007) Interleukin-1receptor antagonist in type 2 diabetes mellitus. N Engl J Med 356:1517-1526

47. Ofei F, Hurel S, Newkirk J, Sopwith M, Taylor R (1996) Effects of an engineered human anti-TNF-alpha antibody (CDP571) on insulin sensitivity and glycemic control in patients with NIDDM. Diabetes 45:881-885

48. Yazdani-Biuki B, Stelzl H, Brezinschek HP et al (2004) Improvement of insulin sensitivity in insulin resistant subjects during prolonged treatment with the anti-TNF-alpha antibody infliximab. Eur J Clin Invest 34:641-642 